\title{
Variantes clínicas infrecuentes del liquen plano: pigmentoso invertido, anular y penfigoide
}

\section{Infrequent clinical variants of lichen planus: pigmentosus-inversus, annular and pemphigoid}

Marina Gagliardi ${ }^{1}$, Corina Busso ${ }^{2}$ y Javier Anaya ${ }^{3}$

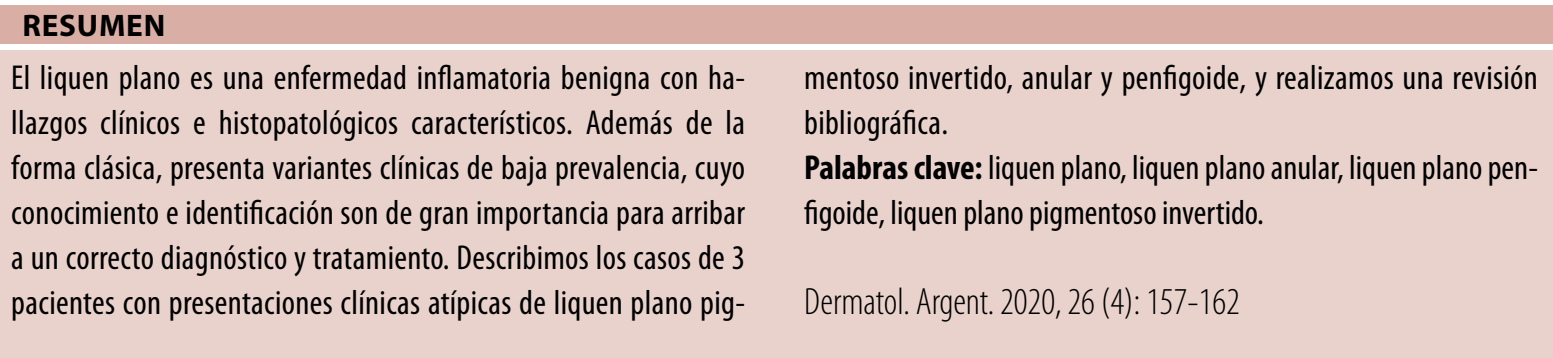

\section{ABSTRACT \\ Lichen planus is a benign inflammatory disease with characteristic clinical and histopathological findings. In addition to the classic form, it presents multiple clinical variants of low prevalence whose knowledge and identification is very important to reach for a correct diagnosis and treatment. We describe three patients with atypical clinical presentations of lichen planus pigmentosus-}

inversus, annular and pemphigoides, and conducted a literature review.

Key words: lichen planus, annular lichen planus, lichen planus pemphigoides, lichen planus pigmentosus-inversus.

Dermatol. Argent. 2020, 26 (4): 157-162
1 Médica de Planta, Servicio de Dermatología

2 Jefa del Servicio de Dermatología

${ }^{3}$ Médico de Planta, Servicio de Anatomía Patológica Hospital Universitario Austral, Universidad Austral, Derqui, Pilar, Provincia de Buenos Aires, Argentina
Contacto del autor: Marina Gagliardi

E-mail:mgagliar@cas.austral.edu.ar Fecha de trabajo recibido: 9/7/2020

Fecha de trabajo aceptado: 28/12/2020

Conflicto de interés: los autores declaran que no existe conflicto de interés.

\section{INTRODUCCIÓN}

El liquen plano (LP) es una dermatosis inflamatoria que puede comprometer la piel, las mucosas y las faneras. Su presentación clínica tiene múltiples variantes: además de su forma clásica -caracterizada por pápulas eritematovioláceas, poligonales, pruriginosas, cubiertas por finas y brillantes escamas blanquecinas denominadas estrías de Wickham- se describen otras formas como anular, pigmentosa invertida, ampollar, penfigoide, hipertrófica, actínica, oral, mucosa, folicular y eritrodérmica. Si bien todas ellas le otorgan al LP una gran heterogeneidad clínica, la histopatología mantiene un patrón básico que permite arribar a un diagnóstico certero $^{1-3}$. Presentamos los casos de 3 pacientes con LP pigmentoso invertido, LP anular y LP penfigoide, y realizamos una revisión bibliográfica.

\section{SERIE DE CASOS}

\section{Caso clínico 1}

Un varón de 42 años consultó por la presencia de máculas amarronadas y rojo-violáceas, de contornos levemente descamativos, localizadas en la región submamaria, el abdomen, la región lumbar, y los pliegues axilar, antecubital e inguinal, levemente pruriginosas, de un mes de evolución (Foto 1). Como antecedente refirió hipotiroidismo de 10 años de evolución, en tratamiento con levotiroxina. Las mucosas y las faneras se encontra- 
ban indemnes. En la dermatoscopia, se observaron áreas violáceas y amarronadas sin estructura, con múltiples puntos marrones (Foto 2). Las pruebas serológicas para las hepatitis $\mathrm{B}$ y $\mathrm{C}$ y para el HIV fueron no reactivas; el perfil tiroideo ( $\mathrm{TSH}, \mathrm{T}_{3} \mathrm{y} \mathrm{T}_{4}$ ) estaba dentro de los límites normales. La anatomía patológica de una muestra de piel de la axila izquierda fue compatible con una dermatitis con patrón inflamatorio liquenoide. Con diagnóstico de liquen plano pigmentoso invertido (LPPI), se indicó tratamiento tópico con clobetasol y tacrolimus al $0,1 \%$ durante 2 meses, con el que se obtuvo una remisión parcial, ya que persistieron las lesiones axilares, y una importante hiperpigmentación posinflamatoria en las otras localizaciones.

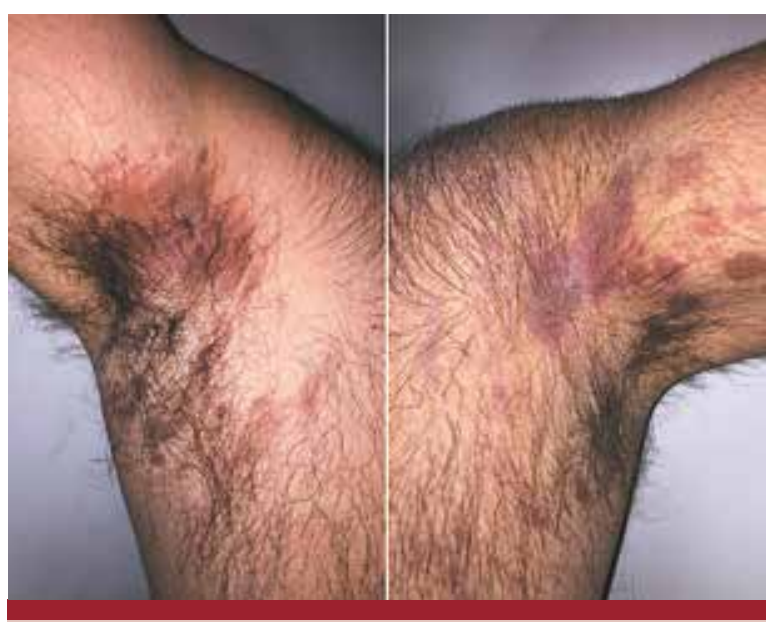

FOTO 1: Máculas amarronadas eritematovioláceas con bordes descamativos en ambas axilas.

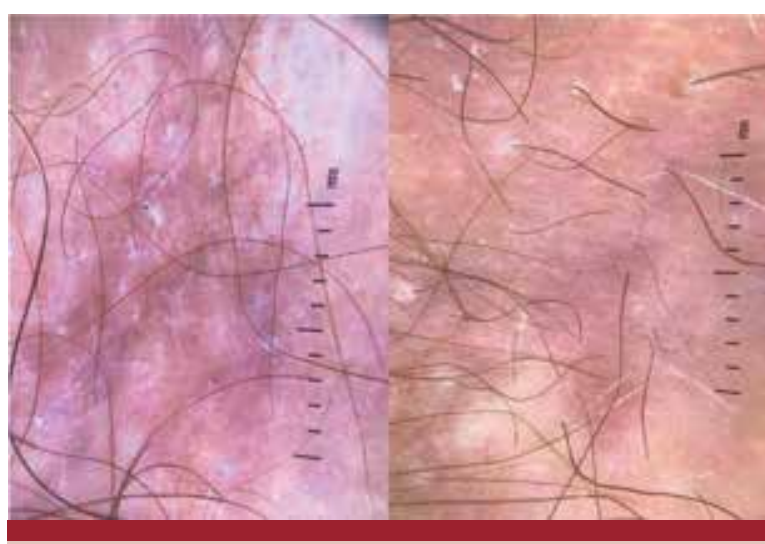

FOTO 2: Dermatoscopia de las lesiones axilares. Se observan puntos marrones que alternan con un fondo de coloración violáceo amarronado sin red.

\section{Caso clínico 2}

Una mujer de 38 ańos, con el antecedente de púrpura trombocitopénica idiopática, consultó por la aparición de lesiones intensamente pruriginosas en el tronco, el pubis, la axila y la rodilla derechas, de un mes de evolución. En el examen físico se evidencia- ban placas de configuración anular, de 0,5 a 1,5 cm de diámetro, con límites eritematosos sobreelevados y centro deprimido hiperpigmentado (Foto 3). Las mucosas y las faneras estaban indemnes. En el laboratorio, se halló una plaquetopenia leve $\left(131.000 / \mathrm{mm}^{3}\right)$, las pruebas serológicas virales fueron no reactivas y el perfil tiroideo era normal. Se realizó una biopsia de la piel del dorso, que fue compatible con una dermatitis liquenoide, por lo que se arribó al diagnóstico de liquen plano anular (LPA). Se indicó tratamiento tópico con clobetasol y antihistamínicos orales por 15 días. Se observó la remisión total del prurito y de las lesiones cutáneas. No se produjo recidiva de la dermatosis durante un año de seguimiento.

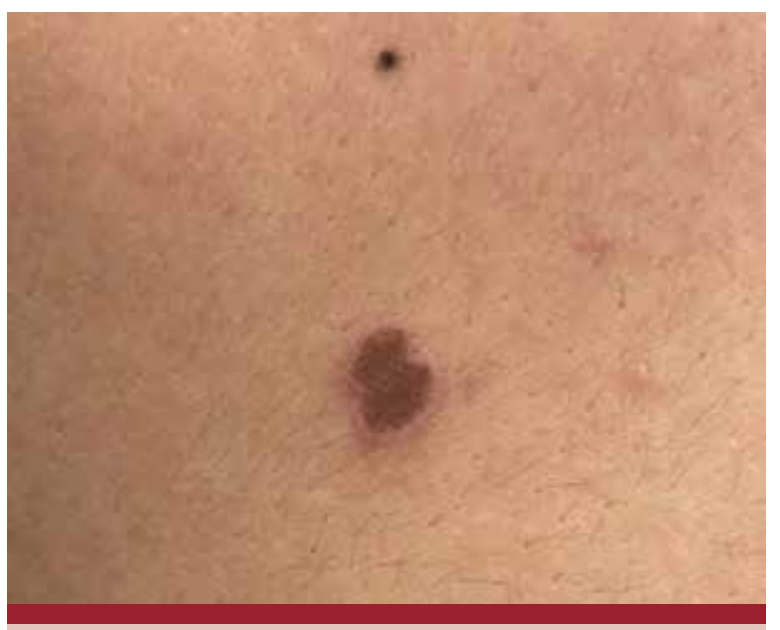

FOTO 3: Placa de configuración anular en el tronco, con límites eritematosos sobreelevados y centro hiperpigmentado.

\section{Caso clínico 3}

Una mujer de 50 ańos, con el antecedente de hipertensión arterial en tratamiento con enalapril, consultó por la presencia de una dermatosis generalizada, muy pruriginosa, de 3 meses de evolución. Se observaban múltiples placas eritematovioláceas de configuración anular, de 0,5 a $2 \mathrm{~cm}$ de diámetro, localizadas en el tronco y los miembros, sobre algunas de las cuales asentaban ampollas tensas de contenido serosanguinolento, que alternaban con erosiones de diferentes tamaños y ampollas sobre piel sana (Fotos 4 y 5). Las mucosas y las faneras no estaban afectadas. Las pruebas serológicas para las hepatitis B y C y para el HIV fueron no reactivas.

En la biopsia de piel de una placa de abdomen se observó despegamiento subepidérmico y, a nivel dérmico, infiltrados linfocitarios superficiales acompañados de melanófagos, hallazgos vinculables a una dermatitis con rasgos liquenoides y despegamiento subepidérmico (Foto 6). El estudio anatomopatológico de una ampolla tensa en el antebrazo derecho fue 


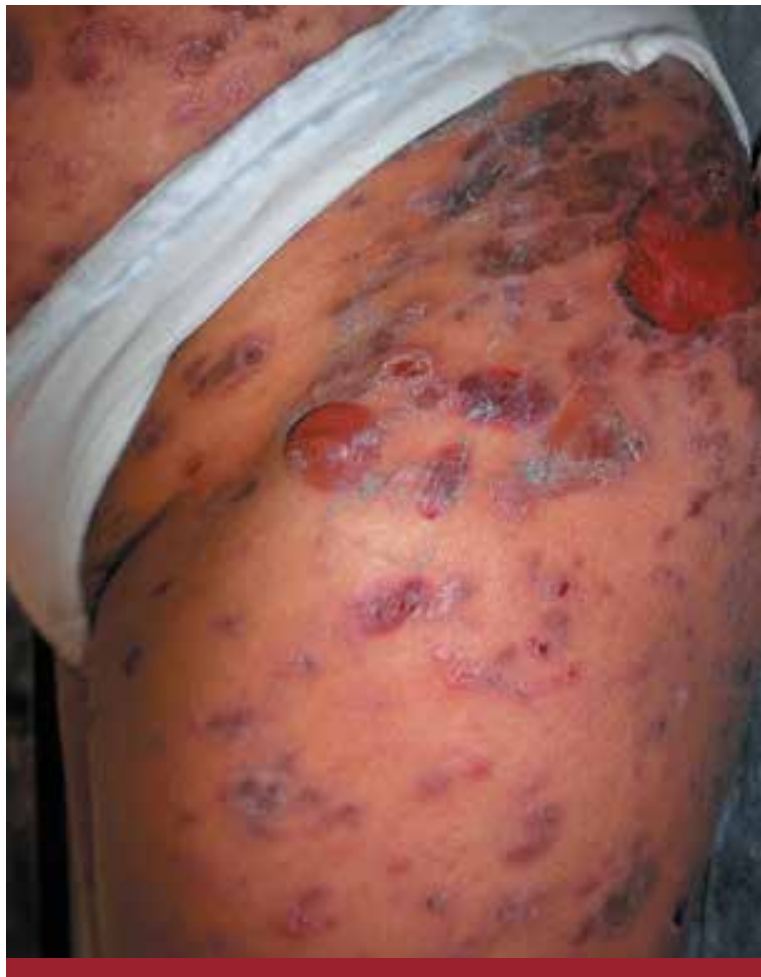

FOTO 4: Múltiples placas eritematovioláceas, sobre algunas asientan ampollas tensas de contenido serosanguinolento, que alternan con erosiones de diferentes tamaños.

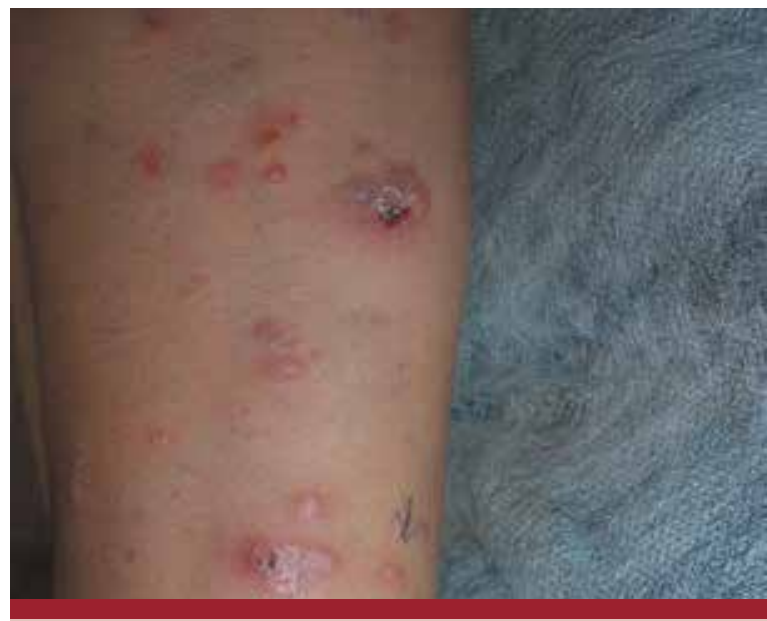

F0T0 5: En el antebrazo, coexistencia de ampollas que asientan tanto sobre piel sana como sobre placas.

compatible con una ampolla subepidérmica con leve eosinofilia. La inmunofluorescencia directa (IFD) de la piel perilesional mostró un depósito lineal de C3 a lo largo de la zona de la membrana basal, negativo para IgG, IgA e IgM. Con los hallazgos clínicos e histológicos, se arribó al diagnóstico de liquen plano penfigoide (LPP) y se indicó tratamiento con meprednisona 40 $\mathrm{mg} /$ día. En un lapso de 20 días, no aparecieron nuevas ampollas y todas las erosiones se habían reepitelizado.

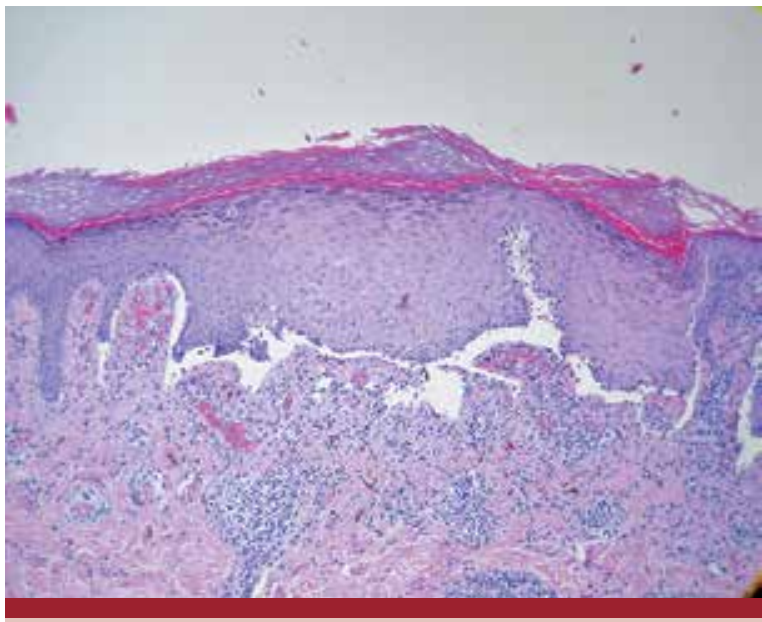

FOTO 6: Se observan hiperqueratosis e hipergranulosis con despegamiento subepidérmico $y$, en la dermis, infiltrados linfocitarios superficiales acompañados de melanófagos. Los hallazgos son vinculables a una dermatitis con rasgos liquenoides y despegamiento subepidérmico (tinción con HyE).

\section{COMENTARIOS}

Las variantes morfológicas pigmentoso invertido, anular y penfigoide del LP son parte de un grupo de formas clínicas infrecuentes que puede adoptar esta dermatosis (Tabla 1). El LPPI, entidad poco frecuente descripta en 2001 por Pock et ál., se caracteriza por la aparición de máculas o placas de límites bien definidos, de coloración marrón violáceo, levemente pruriginosas o asintomáticas que se localizan preferentemente en zonas no fotoexpuestas, flexuras y áreas intertriginosas: axilas en el 90\% de los casos, ingles, región submamaria, huecos poplíteos y pliegues retroauriculares ${ }^{4-6}$. Estas localizaciones permiten diferenciarlo del LP pigmentoso (LPpi), cuyas lesiones son similares, pero distribuidas en áreas fotoexpuestas ${ }^{4}$. En el 10\% de los casos, el LPPI puede coexistir con lesiones de LP clásico o $\mathrm{LPpi}^{7}{ }^{7,}$. Las uñas y las mucosas permanecen indemnes.

En la dermatoscopia se pueden observar tres patrones: 1) difuso, relacionado con la pigmentación de la epidermis; 2) puntos azules, grises o amarronados, que representan los melanófagos dérmicos; 3) mixto. Estos hallazgos dermatoscópicos tienen valor pronóstico, ya que el patrón de puntos implica una mayor profundidad del pigmento $y$, por ende, una dermatosis más persistente ${ }^{6,7,9}$. El paciente del caso 1 presentaba un patrón mixto y su evolución clínica coincidía con los datos publicados de otros casos.

La histopatología muestra vacuolización de la capa basal, como ocurre en el LP clásico, pero se diferencia de este por la presencia de atrofia epidérmica y la ausencia de acantosis reactiva. Esto parece obedecer a una intensa reacción liquenoide producida en un corto período, lo que da lugar a una importante vacuolización 


\begin{tabular}{|c|c|c|c|}
\hline & LP pigmentoso invertido & LP anular & LP penfigoide \\
\hline Edad y sexo & Adultos, sin predilección por sexo & $\begin{array}{l}\text { Adultos, ligero predominio en } \\
\text { hombres }\end{array}$ & $\begin{array}{l}\text { Adultos (pico incidencia entre los } 50 \text { y } \\
60 \text { años), leve predominio en mujeres }\end{array}$ \\
\hline $\begin{array}{l}\text { Examen } \\
\text { físico }\end{array}$ & $\begin{array}{l}\text { Máculas o placas de límites bien } \\
\text { definidos, de coloración marrón violá- } \\
\text { cea, pruriginosas o asintomáticas } \\
\text { No presenta compromiso de mucosas } \\
\text { ni faneras }\end{array}$ & $\begin{array}{l}\text { Máculas o placas eritematovioláceas } \\
\text { anulares, de contornos sobreelevados } \\
\text { y centro atrófico, asintomáticas o } \\
\text { pruriginosas } \\
\text { Puede existir compromiso mucoso, } \\
\text { ungueal y del cuero cabelludo }\end{array}$ & $\begin{array}{l}\text { Pápulas típicas de LP que coexisten con } \\
\text { ampollas tensas que asientan sobre } \\
\text { dichas lesiones y sobre piel sana }\end{array}$ \\
\hline Localización & $\begin{array}{l}\text { Grandes pliegues y zonas no fotoex- } \\
\text { puestas }\end{array}$ & $\begin{array}{l}\text { Formas localizadas: pene y escroto, } \\
\text { grandes pliegues } \\
\text { Existen formas generalizadas }\end{array}$ & Miembros superiores e inferiores \\
\hline $\begin{array}{l}\text { Asocia- } \\
\text { ciones }\end{array}$ & Hepatitis C & $\begin{array}{l}\text { Hepatitis B y C, hepatitis autoinmuni- } \\
\text { taria, cirrosis biliar primaria }\end{array}$ & $\begin{array}{l}\text { Fármacos: inhibidores de la ECA } \\
\text { (enalapril, captopril, ramipril), sim- } \\
\text { vastatina, antifímicos, cinarizina y } \\
\text { pembrolizumab } \\
\text { Neoplasias: liposarcoma retroperi- } \\
\text { toneal, leucemia linfocítica crónica y } \\
\text { adenocarcinoma de colon } \\
\text { PUVA } \\
\text { Enfermedad de Castleman }\end{array}$ \\
\hline $\begin{array}{l}\text { Estudio } \\
\text { histológico }\end{array}$ & $\begin{array}{l}\text { Infiltrado inflamatorio liquenoide con } \\
\text { vacuolización de la capa basal asociado } \\
\text { a atrofia epidérmica con ausencia de } \\
\text { acantosis reactiva } \\
\text { En la dermis papilar el infiltrado } \\
\text { inflamatorio alterna con importante } \\
\text { melanofagia e incontinencia del } \\
\text { pigmento }\end{array}$ & $\begin{array}{l}\text { En el borde de las lesiones: cambios } \\
\text { característicos de LP, mientras que } \\
\text { en el centro se observa un infiltrado } \\
\text { linfocitario disperso y con menor } \\
\text { número de células de Langerhans }\end{array}$ & $\begin{array}{l}\text { Pápula: cambios típicos de LP } \\
\text { Ampolla: despegamiento ampollar } \\
\text { subepidérmico con un infiltrado } \\
\text { variable de linfocitos y eosinófilos, con } \\
\text { neutrófilos o sin ellos, en la dermis } \\
\text { superior }\end{array}$ \\
\hline IFD e IFI & & & $\begin{array}{l}\text { IFD: C3 solo o acompañado de lgG con } \\
\text { disposición lineal a lo largo de la unión } \\
\text { dermoepidérmica } \\
\text { IFI: autoanticuerpos circulantes que } \\
\text { se unen al colágeno de tipo XVII. Con } \\
\text { técnica de salt split, los anticuerpos se } \\
\text { unirán al techo de la ampolla }\end{array}$ \\
\hline Tratamiento & Clobetasol y tacrolimus al $0,1 \%$ & $\begin{array}{l}\text { Tópico: clobetasol y tacrolimus al } \\
0,1 \% \\
\text { Sistémico: fototerapia, corticosteroi- } \\
\text { des orales asociados a hidroxicloro- } \\
\text { quina o dapsona }\end{array}$ & $\begin{array}{l}\text { De elección: corticosteroides, en forma } \\
\text { tópica en los casos leves y por vía } \\
\text { sistémica en los más severos } \\
\text { Otras opciones: isotretinoína, dapsona, } \\
\text { tetraciclinas, nicotinamida, ciclospori- } \\
\text { na, metotrexato y ustekinumab }\end{array}$ \\
\hline Evolución & $\begin{array}{l}\text { Variable. Desde autoinvolución en } \\
\text { pocas semanas a casos recalcitrantes a } \\
\text { múltiples tratamientos tópicos }\end{array}$ & $\begin{array}{l}\text { Buena respuesta al tratamiento } \\
\text { Mayor dificultad en casos generali- } \\
\text { zados }\end{array}$ & $\begin{array}{l}\text { Buena. Tiende a tener un curso más } \\
\text { localizado y benigno que otras derma- } \\
\text { tosis ampollares }\end{array}$ \\
\hline
\end{tabular}

TABLA 1: Características clínico-patológicas del liquen pigmentoso invertido, el liquen plano anular y el liquen plano penfigoide. 
de la capa basal sin la acantosis compensatoria observada en el LP clásico. Luego, una rápida regresión del infiltrado inflamatorio daría como resultado una epidermis atrófica con signos de melanofagia e incontinencia de pigmento en la dermis ${ }^{4}$.

Como desencadenantes de la dermatosis, se plantean estímulos externos como la fricción y el uso de ropa ajustada, que pueden generar un fenómeno de Köebner $^{4,6,10}$. Entre los diagnósticos diferenciales se destacan el LPpi y el LP actínico, ambos con localización en zonas fotoexpuestas y de predominio en personas con fototipo alto. También se debe diferenciar de otras dermatosis que asientan en los pliegues, como el eritema fijo pigmentado medicamentoso, el intertrigo candidiásico, la acantosis nigricans, las farmacodermias liquenoides, las dermatitis por contacto pigmentadas y la dermatosis cenicienta ${ }^{8,9}$.

Se describió el LPPI en asociación con fármacos antihipertensivos (como en la última paciente), infección por hepatitis $\mathrm{C}$ y exposición laboral a metales pesados en un trabajador de una fábrica de semiconductores $^{8,10-12}$. No se han comunicado asociaciones con neoplasias. En cuanto al antecedente de hipotiroidismo del paciente del caso 1, resulta difícil relacionarlo con la dermatosis, dado que hacía 10 años que seguía el tratamiento y su patología de base estaba controlada. El tratamiento de elección del LPPI es tópico, con corticosteroides de alta potencia (propionato de clobetasol) y tacrolimus al 0,1\%. Debido al carácter autoinvolutivo y benigno de la dermatosis, se desaconsejan tratamientos más agresivos ${ }^{8,9}$.

El 7-10\% de los pacientes con LP presentan la variedad anular, que se manifiesta con máculas o placas eritematovioláceas anulares, de contornos o límites sobreelevados y centro atrófico, asintomáticas o pruriginosas $^{3}$. La localización genital, en especial en el pene y el escroto, es frecuente. También se describe en los grandes pliegues como axilas e ingles, el tronco, las extremidades y se ha publicado un caso con afección de la semimucosa labial ${ }^{3,13,14}$. Si bien puede presentarse de forma generalizada, predomina la variedad localizada ${ }^{3}$. Esta última puede ser indistinguible clínicamente del granuloma anular o la poroqueratosis de Mibelli, por lo que la histopatología es indispensable para su diferenciación ${ }^{15,16}$. Se encuentran publicaciones en donde se relaciona el liquen plano anular con las hepatitis $\mathrm{B}$ y $\mathrm{C}$, la hepatitis autoinmunitaria y la cirrosis biliar primaria, pero no hay evidencia de asociación con la púrpura trombocitopénica idiopática como presentaba la paciente, por lo que queda abierto el interrogante acerca del valor de este antecedente en la aparición de la dermatosis ${ }^{15}$. El tratamiento indicado son los corti- costeroides tópicos, en las lesiones localizadas y por vía sistémica, asociados a hidroxicloroquina o dapsona, en las formas generalizadas ${ }^{3}$.

El LPP es una dermatosis ampollar subepidérmica cuya etiopatogenia no está totalmente aclarada, ya que podría ser una variante propia del LP o la presentación concomitante de dos enfermedades: el LP y el penfigoide ampollar $(\mathrm{PA})^{17,18}$. En este sentido, la teoría con mayor consenso sobre la fisiopatogenia del LPP es la de expansión de epítopos: en el LP se dañarían los queratinocitos basales, con la consecuente exposición de nuevos determinantes antigénicos que antes permanecían ocultos y desencadenarían una respuesta inmune con formación de anticuerpos y activación del complemento, igual a la observada en el $\mathrm{PA}^{17,19}$. Un artículo publicado por Hübner et ál. aporta evidencia para considerar el LPP una enfermedad ampollar subepidérmica autoinmune asociada a cambios liquenoides ${ }^{20}$. Esos autores plantean que, dado que en la mayoría de los casos las lesiones cutáneas liquenoides preceden a la formación de ampollas, la inflamación mediada por células $T$ puede disparar una respuesta autoinmune contra proteínas de la unión dermoepidérmica. En el LPP predominan los autoanticuerpos contra el colágeno de tipo XVII, una proteína de $180 \mathrm{kDA}$ expresada en los hemidesmosomas de la unión dermoepidérmica, también involucrada en la fisiopatogenia del PA, el penfigoide de las mucosas y la dermatosis por IgA lineal. Mediante el uso de fragmentos recombinantes del subdominio NC16A del colágeno de tipo XVII, se demostró que en los pacientes con LPP hay diferencias sutiles en la especificidad del epítopo, lo cual permite diferenciarlo de las otras dermatosis mencionadas. También se encontraron otros autoanticuerpos contra proteínas desconocidas de $130 \mathrm{kDa}$ y $200 \mathrm{kDa}$. Estos hallazgos indicarían un patrón inmunitario propio del LPP, que permitiría considerarlo dentro de una amplia familia de enfermedades caracterizadas por autoanticuerpos contra el colágeno de tipo XVII ${ }^{20}$.

Esta forma clínica presenta pápulas de LP que alternan con ampollas tensas, localizadas sobre esas lesiones y también en piel sana, con predilección por las extremidades. Predomina en los adultos, con un pico de prevalencia entre los 50 y los 60 años, como ocurrió en el caso analizado ${ }^{3,17,20}$. Los hallazgos histológicos y de IFD dependerán de la lesión estudiada. En la pápula se observan los cambios típicos de LP y la IFD muestra el depósito de IgM y C3 en los cuerpos coloides, la fibrina y el fibrinógeno, mientras que en la ampolla se evidencia un despegamiento ampollar subepidérmico con un infiltrado variable de linfocitos y eosinófilos, con neutrófilos o sin ellos, en la dermis superior. En la 
IFD, la presencia de C3, solo o acompañado de IgG con disposición lineal a lo largo de la unión dermoepidérmica, permite confirmar el diagnóstico ${ }^{17-20}$. A través de métodos como ELISA, immunoblotting o inmunofluorescencia indirecta (IFI), se pueden detectar los autoanticuerpos circulantes en el suero que se unen a los dominios NC16A o C-terminales del colágeno de tipo XVII. En la IFI con técnica de salt split, los anticuerpos se unen al techo de la ampolla ${ }^{20}$. El principal diagnóstico diferencial debe plantearse con el LP ampollar, en el que las ampollas se distribuyen exclusivamente sobre las pápulas, como consecuencia de la rotura dermoepidérmica provocada por el intenso y rápido infiltrado inflamatorio a ese nivel. Dado el mecanismo de formación, en el LP ampollar no se evidencia el depósito de anticuerpos ni de complemento en la IFD ${ }^{17,20}$. También debe diferenciarse del PA: el LPP tiende a ser más localizado, de curso más benigno y los pacientes suelen ser más jóvenes que los afectados por el primero ${ }^{21}$. El LPP en general es idiopático, pero se han publicado casos asociados a fármacos como enalapril, captopril, ramipril, simvastatina, antifímicos, cinarizina y pembrolizumab ${ }^{17-20}$. Para poder considerar

\section{BIBLIOGRAFÍA}

1. Tziotzios C, Lee JYW, Brier T, Saito R, et ál. Lichen planus and lichenoid dermatoses: Clinical overview and molecular basis. J Am Acad Dermatol 2018;79:789-804.

2. Le Cleach L, Chosidow O. Clinical practice. Lichen planus. $N$ Engl J Med 2012;366:723-732.

3. Weston G, Payette M. Update on lichen planus and its clinical variants. Int J Womens Dermatol 2015;1:140-149.

4. Pock L, Jelínková L, Drlík S, Abrhámová S, et ál. Lichen planus pigmentosus-inversus. J Eur Acad Dermatol Venereol 2001;15:452-454.

5. Mohamed M, Korbi M, Hammedi F, Youssef $M$, et ál. Lichen planus pigmentosus inversus: a series of 10 Tunisian patients. Int J Dermatol 2016;55:1088-1091.

6. Peralta R, Pazos $M$, Cohen Sabban E, Schroh $R$, et ál. Liquen plano pigmentoso invertido. Arch Argent Dermatol 2015;65:189-194.

7. Imbernón-Moya A, Churruca-Grijelmo $M$, Martínez-Pérez M, Lobato-Berezo A. Dermatoscopia del liquen plano pigmentado inverso. Actas Dermosifiliogr 2015;106:857-859.

8. Bennàssar A, Mas A, Julià M, Iranzo $P$, et ál. Placas anulares en grandes pliegues: cuatro casos de liquen plano pigmentosoinverso. Actas Dermosifiliogr 2009;100:602-605.

9. Rodríguez AM, Jurado SF, González GM, Alfaro OLP, et ál. Liquen plano pigmentado invertido: revisión del tema a propósito de un caso. Rev Cent Dermatol Pascua 2013;22:115-119.

10. Ryynänen AT, Von Willebrand M, Kluger N. Lichen planus pigmentosus-inversus in a Finnish man. J Eur Acad Dermatol Venereol 2019;33:e64-e65. una asociación causal con un medicamento, es necesario que este se haya incorporado poco tiempo antes de la aparición del LPP: en el caso 3, la paciente se encontraba en tratamiento con enalapril desde hacía 4 años, por lo que se aleja esta posibilidad ${ }^{20}$. También se lo asoció a PUVA, enfermedad de Castleman y neoplasias malignas como liposarcoma retroperitoneal, leucemia linfocítica crónica y adenocarcinoma de colon $^{3,17-20}$. Con respecto al tratamiento, el LPP suele tener buena respuesta a los glucocorticoides. Se administran en forma tópica en los casos leves y por vía sistémica en los más severos. Otras opciones terapéuticas son: isotretinoína, dapsona, tetraciclinas, nicotinamida, ciclosporina, metotrexato $y$, recientemente, se obtuvo buena respuesta en un paciente tratado con ustekinumab ${ }^{18}$.

Presentamos tres variantes clínicas poco frecuentes de LP que suscitaron un desafío diagnóstico (Tabla 1). Nuestro caso de LPPI, según la búsqueda bibliográfica realizada hasta la fecha, sería el tercer caso publicado en Argentina y el 63 en la literatura médica mundial. Asimismo, destacamos el gran espectro clínico que presenta el LP y la importancia de la histopatología, herramienta crucial para arribar al diagnóstico de certeza.

11. Matamoros E, Michelena MA, Campoy MV, Teberobsky MV, et ál. Liquen plano pigmentoso invertido asociado a virus de hepatitis C. Arch Argent Dermatol 2013;63:55-58.

12. Hsu CY, Liu D, Lee WR, Shih YH. Lichen planus pigmentosus inversus caused by occupational systemic sensitization to metals in a semiconductor factory worker. Dermatitis 2017;28:324-326.

13. Reich HL, Nguyen JT, James WD. Annular lichen planus: a case series of 20 patients. J Am Acad Dermatol 2004;50:595-599.

14. Holmukhe S, Gutte RM, Sirur S. Letter: Isolated annular lichen planus of lower lip. Dermatol Online J 2012;18:15.

15. Andreone RA, Fandiño M, Turner V, Maronna E. Lesiones anulares generalizadas. Dermatol Argent 2016;22:214-216.

16. Chakraborty S, Chowdhury J, De A, Gharami RC. Generalized annular lichen planus with a unique morphology in a patient seropositive for HIV. JAAD Case Rep 2015;1:251-253.

17. De Diego MC, Eimer L, Suar L, Marchese ML, et al. Liquen plano con ampollas: piense en liquen plano penfigoide. Dermatol Argent 2013;19:286-288.

18. Knisley RR, Petropolis AA, Mackey VT. Lichen planus pemphigoides treated with ustekinumab. Cutis 2017;100:415-418.

19. Onprasert W, Chanprapaph K. Lichen Planus Pemphigoides Induced by Enalapril: A Case Report and a Review of Literature. Case Rep Dermatol 2017;9:217-224.

20. Hübner F, Langan EA, Recke A. Lichen Planus Pemphigoides: From Lichenoid Inflammation to Autoantibody-Mediated Blistering. Front Immunol 2019;10:1389.

21. Wagner G, Rose C, Sachse MM. Clinical variants of lichen planus. J Dtsch Dermatol Ges 2013;11:309-319. 